\title{
PARALISIA CEREBRAL: ANÁLISE DOS PADRÕES \\ DA DEGLUTIÇÃO ANTES E APÓS \\ INTERVENÇÃO FONOAUDIOLÓGICA
}

\author{
Cerebral palsy: analysis of swallowing patterns \\ before and after speech therapy intervention
}

Cláudia Inês Oliveira Vianna ${ }^{(1)}$, Heloisa Sawada Suzuki (2)

\begin{abstract}
RESUMO
Objetivo: analisar os padrões da deglutição antes e após intervenção fonoaudiológica em um grupo de crianças com Paralisia Cerebral que apresentam quadro de Disfagia. Método: estudo longitudinal, constituído por vinte crianças portadoras de Paralisia Cerebral, com idades entre um e oito anos, de ambos os sexos. Foi utilizada anamnese com os responsáveis e os pacientes foram submetidos à avaliação funcional da deglutição antes e após intervenção fonoaudiológica num período de três meses. Resultados: percebeu-se discreta melhora no padrão de deglutição o que consequentemente trouxe um melhor padrão de alimentação aos pacientes avaliados e orientados. Conclusão: o presente estudo mostrou que a intervenção fonoaudiológica aos pacientes portadores de Paralisia Cerebral traz como resultado melhorias em seu padrão de alimentação, apesar do curto período de atendimento (três meses).
\end{abstract}

DESCRITORES: Paralisia Cerebral; Fonoterapia; Alimentação; Crianças

\section{INTRODUÇÃO}

O termo "Paralisia Cerebral" (PC) engloba um grande espectro de entidades clínicas que se manifestam por uma desordem no desenvolvimento motor, amplamente variável em etiologia, manifestações, gravidade, prognóstico e comorbidade. $\mathrm{Na}$ verdade, trata-se de um complexo de sintomas mais do que uma doença específica, cujo denominador comum é o fato de ser decorrente de lesão não progressiva do Sistema Nervoso Central imaturo. O evento lesivo pode ocorrer no período pré, peri ou pós-natal ${ }^{1}$.

A maior causa de PC no nosso meio é anóxia perinatal por um trabalho de parto anormal ou prolongado. A prematuridade é a segunda maior causa. Com menor freqüência estão infecções

(1) Fonoaudióloga da Associação Brasileira Beneficente de Reabilitação - ABBR; Pós- Graduada em Educação Psicomotora e Fonoaudiologia Hospitalar; Especialista em Motricidade Orofacial.

(2) Fonoaudióloga; Doutora em Ciências pela Universidade Federal do Estado de São Paulo - Unifesp/EPM.

Conflito de interesses: inexistente pré-natais, como rubéola, toxoplasmose, citomegalovírus e as infecções pós-natais como as meningites ${ }^{2}$. Essa casuística é confirmada nos pacientes em atendimento na Associação Brasileira Beneficente de Reabilitação (ABBR).

Fica muito evidente a importância da vigilância fetal, se houver evidências de déficit de oxigenação no nascimento ${ }^{3}$, para que essa criança seja encaminhada para tratamento de reabilitação o mais breve possível, minimizando assim, futuras possíveis sequelas.

Os pacientes com PC sofrem diminuição da oxigenação cerebral, lesando áreas do controle da deglutição ${ }^{4}$. A deglutição acontece como um processo contínuo. Distúrbios no processo da deglutição podem causar problemas na alimentação, sendo estes transtornos chamados de disfagia ${ }^{5-7}$.

As alterações observadas na disfagia orofaríngea neurogênica incluem: dificuldade em iniciar a deglutição, inadequação da sensibilidade e mobilidade de estruturas da cavidade oral, incoordenação no controle da língua, sialorréia, regurgitação nasal, ineficiência na mobilidade dos músculos laríngeos e episódios de engasgo e tosse durante a 
alimentação ${ }^{8}$. E podem causar complicações como a desnutrição, a desidratação e complicações respiratórias $^{9}$. A aspiração traqueal, ou seja, entrada de saliva ou alimentos nas vias aéreas pode acarretar infecções recorrentes como a pneumonia aspirativa. Esta intercorrência clínica pode levar os pacientes à internação, interrompendo, desta forma, a programação terapêutica.

Nos casos em que o comprometimento neurológico é leve, observa-se que os distúrbios motores orais geralmente são restritos à fase oral da deglutição, sem impacto no quadro clínico geral. Assim, a reabilitação dos padrões funcionais, necessários tanto para fala quanto para deglutição, é mais facilmente alcançada. Porém, quando o comprometimento é moderado ou severo e há intensas alterações motoras, globais e orais muitas vezes associadas à presença de múltiplas deficiências, instala-se um quadro de disfagia, somado às alterações da patologia ${ }^{10}$.

$\mathrm{Na}$ tentativa de evitar comprometimentos clínicos e nutricionais, as crianças com paralisia cerebral que apresentam alterações na deglutição são encaminhadas para terapia fonoaudiológica em disfagia, com o objetivo de promover uma maior funcionalidade na alimentação e de verificar se a via de alimentação utilizada encontra-se segura e eficiente para cada criança ${ }^{11}$.

O fonoaudiólogo, em sua avaliação, deve estar atento a todos os músculos e funções orofaciais $^{12}$. Não deixando de orientar também quanto à postura e ao posicionamento do paciente durante a avaliação.

O objetivo deste trabalho é analisar os padrões da deglutição antes e após intervenção fonoaudiológica em crianças com Paralisia Cerebral que apresentam quadro de disfagia.

\section{MÉTODO}

Estudo longitudinal realizado com vinte crianças com diagnóstico médico de paralisia cerebral com diferentes classificações do quadro motor, na faixa etária de um a oito anos, de ambos os sexos, que fazem atendimento multidisciplinar no Setor Infanto Juvenil da Associação Brasileira Beneficente de Reabilitação - ABBR - na cidade do Rio de Janeiro, no período de setembro a dezembro de 2007. Dez pacientes eram do sexo feminino $(50 \%)$ e dez do sexo masculino (50\%). A participação somente foi considerada após concordância com o termo de consentimento livre e esclarecido por parte dos responsáveis.

Os atendimentos foram realizados em grupo e cada grupo era composto por três crianças e seus respectivos cuidadores. As sessões de atendimento tiveram a duração de vinte e cinco minutos. Foi acordado, previamente, que não haveria troca de cuidadores durante a pesquisa, para que as orientações transmitidas aos mesmos pela fonoaudióloga pudessem ser seguidas ao longo da pesquisa.

$\mathrm{Na}$ primeira fase do trabalho foram realizadas anamneses com os responsáveis investigando as queixas relacionadas a dificuldades na alimentação e/ou deglutição da criança, desde o período de amamentação natural e/ou amamentação artificial até o período de introdução de outros alimentos. Observou-se também se a criança apresentava crises convulsivas, refluxo gastroesofágico, a medicação utilizada e a via de alimentação (via oral ou via alternativa).

Na segunda fase do trabalho os pacientes foram submetidos à avaliação funcional da deglutição, isto é, foi observado no momento da captação do alimento se houve o vedamento labial e a protrusão de língua. Outros aspectos também foram observados além do vedamento labial e da protrusão de língua. Observou-se a regurgitação nasal do alimento e escape extra-oral do mesmo. A ejeção oral também foi observada assim como munching. Verificou-se ainda se havia presença de sinais sugestivos de aspiração, como cianose, cansaço após alimentação, engasgos durante e após oferta dos alimentos e presença de tosse.

As orientações para realização de manobras fonoaudiológicas para adequação dos padrões da deglutição, bem como do posicionamento correto da criança foram sempre esclarecidas aos cuidadores, incluindo neste ítem a modificação e alteração das consistências dos alimentos para que essas fossem seguras para as crianças.

As posturas orientadas foram de acordo com a idade e a gravidade de cada caso. Visavam à inibição de padrões reflexos patológicos, de modo a tornar possíveis os movimentos isolados dos braços, cabeça, mandíbula, língua e lábios. A postura do cuidador é muito importante, porque ele deve controlar toda a movimentação geral da criança e ficar com o tronco e braços livres para manipular colher, copo, e etc.

A alimentação oferecida era trazida pelos responsáveis nas consistências solicitadas para avaliação. Como consistência líquida foram utilizados refresco de frutas e água. Como pastosa, foi utilizado iogurte com consistência mais firme.

A função de mastigação não é eficiente nestas crianças, fazendo com que estes alimentos sejam deglutidos inteiros, ou mesmo aumentando o tempo de manipulação oral do bolo, deixando a refeição com maior gasto energético ${ }^{11}$. Dessa maneira, a alimentação na consistência sólida não foi considerada e descrita nessa pesquisa, pois dos vinte 
pacientes avaliados apenas cinco conseguiam ingerir alimentos sólidos sem apresentar risco de aspiração traqueal ou estase em recessos faríngeos.

Em alguns casos trocas e substituições de utensílios foram necessárias, como por exemplo, material de aço inoxidável (colher) para material plástico resistente ou silicone.

Foram orientados também exercícios miofuncionais para melhor adequação das estruturas envolvidas no processo de deglutição, e manobras facilitadoras para adequação do padrão de deglutição, como por exemplo, manobra para vedamento labial.

A anamnese e avaliação funcional foram adaptadas do protocolo de entrevista inicial e avaliação elaborado por Furkim, Henrique e Frazão 10 (Figura 1).

Este projeto foi aprovado pelo Comitê de Ética em pesquisa com Seres Humanos do CEFAC Centro de Especialização em Fonoaudiologia Clínica, sob o número 138/07.

Foi realizada análise estatística descritiva dos dados obtidos na avaliação realizada antes e após fonoterapia.

\section{RESULTADOS}

As vinte crianças avaliadas alimentavam-se exclusivamente por via oral, ingerindo alimentos líquidos e pastosos.

Após avaliação das vinte crianças, foi montado um plano de atendimento para cada paciente. Seus cuidadores foram orientados quanto à postura adequada para o momento da alimentação e após esse momento, como por exemplo, promover o alinhamento biomecânico das estruturas orais para que a alimentação ocorresse de forma mais próxima ao normal e natural, a respeitar o tempo de refeição do paciente, que o ambiente estivesse calmo, utilização de consistências mais seguras para deglutição, troca de utensílios inadequados.
Em relação a outros tipos de postura e posicionamento, manobras adequadas, exercícios miofuncionais e massagens peri e intra-orais, os mesmos encontram-se descritos na literatura ${ }^{13-15}$.

Com relação à alimentação líquida antes da fonoterapia, tanto na captação do alimento quanto na deglutição do mesmo, a oclusão dos lábios (35\%) e a protrusão da língua (70\%) mantiveram a mesma proporção.

A presença de tosse aconteceu em dez pacientes (50\%), e esse dado foi observado sempre durante a ingesta do alimento líquido. Não foi observada a presença de tosse nem antes, nem após alimentação com líquidos.

Os cuidadores ofertaram refresco de frutas ou água. Sendo oferecido de acordo com a preferência da criança, não alterando significativamente a ingesta de líquidos, nem os resultados obtidos (Tabela 1).

Em relação a alimentos pastosos oferecidos na colher foi percebida uma pequena diferença na captação do bolo, antes da fonoterapia, os pacientes apresentavam $65 \%$ de alteração na oclusão dos lábios. Esse dado diminuiu para 50\% dos casos após fonoterapia (Tabela 2).

Foram mantidos, na mesma proporção, os padrões antes e após fonoterapia em alimentos líquidos. Doze pacientes (60\%) mantiveram ejeção oral adequada. Em treze pacientes (65\%) não houve mudança no padrão munching.

Em relação a alimentos pastosos, a ejeção oral adequada foi mantida em doze pacientes avaliados $(60 \%)$, mas onze pacientes (55\%) ainda apresentavam munching na hora da ingesta (Tabela 3).

Observou-se que após a fonoterapia o percentual de melhora no padrão de alimentação desses pacientes apresentou-se um pouco mais eficaz de acordo com o resultado obtido (Tabela 4).

É importante salientar que nas duas consistências avaliadas houve diminuição dos sinais clínicos de aspiração (70\% em líquido e $75 \%$ em pastoso). $\mathrm{O}$ que diminuiu também as faltas ao atendimento devido a pneumonias aspirativas. 


\section{PROTOCOLO DE ENTREVISTA INICIAL E AVALIAÇÃO}

1.Nome:

Data:

Entrevista respondida por:

D.N:

Diagnóstico Médico:

2. Histórico:

Queixa:

Você percebe que seu filho tem alguma alteração na deglutição: $\quad S(\quad) \quad N(\quad)$

Amamentação Natural:

Sugou no seio? S( ) N ( ) Quando sugou pela 1 $1^{\mathrm{a}} \mathrm{vez}$ ?

Sugou bem? S( ) N( )

Até quando?

Regurgitação Nasal S（） N( )

Escape extra-oral $\mathrm{S}(\quad) \mathrm{N}(\quad)$

Engasgos $\mathrm{S}(\quad) \mathrm{N}(\quad)$

Tosse $\mathrm{S}(\quad) \mathrm{N}(\quad)$

OBS:

\section{Amamentação Artificial:}

Quando foi introduzida a mamadeira? Aceitação: S( ) N( )

Bico utilizado: ortodôntico S ( ) N ( )

Tipo de furo: convencional $\mathrm{S}(\quad) \quad \mathrm{N}(\quad)$ aumentado $\mathrm{S}(\quad) \mathrm{N}(\quad)$ adequado p/ consistência $\mathrm{S}(\quad) \mathrm{N}(\quad)$ Tempo: Posição

Regurgitação Nasal S ( ) N ( )

Engasgos $\mathrm{S}(\quad) \mathrm{N}(\quad)$

Escape extra-oral $\mathrm{S}(\quad) \mathrm{N}(\quad)$

OBS:

Introdução de outros alimentos:

Quando iniciou alimentação na colher?

Tipo de colher: Chá ( ) Sobremesa ( ) Sopa ( ) Metal ( ) Plástica ( )

Aceitação:

Adequada

Rejeição

Alimentos pastosos salgados

Macios em pedaços

Sólidos

Regurgitação Nasal S（） N （）

Tosse $\mathrm{S}(\quad) \mathrm{N}(\quad)$

Engasgos S( ) N( )

Tosse $\mathrm{S}(\mathrm{N}) \mathrm{N}(\mathrm{)}$

Dificuldade com algum alimento? S ( ) N ( ) - Qual?

OBS:

3. Crises Convulsivas? S( ) N( )

4. Apresenta RGE? S( ) N( )

5. Medicação Atual:

\section{Nutrição}

Via oral

Dieta Parcial: $\%$ via oral $\%$ via alternativa

Via oral só para estimulação: Quantidade:

Consistência: Líquida ( ) Pastosa ( ) Sólida ( )

Reações durante a alimentação atual:

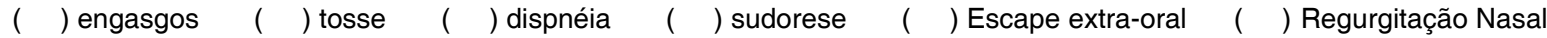
Dificuldade em algum alimento S ( ) N ( ) - Qual? 
Engole pedaços sem mastigar S( ) N ( )

Resíduos de alimentos na cavidade oral após deglutição: $S(\quad) \quad N(\quad)$

Atitudes facilitadoras: oferta de líquido S ( ) N ( ) quantidade: Peq ( ) Grande ( ) Ritmo:lento ( ) rápido ( ) Utensílios Utilizados:

Líquido Pastoso Sólido

Via alternativa de alimentação:

Tipo de dieta: ( ) caseira $\quad(\quad)$ industrializada

Tipo de Sonda:

AVALIAÇÃO FUNCIONAL DA DEGLUTIC̣ÃO:

LÍQUIDO:

- Captação:

- lábios ocluídos $\mathrm{S}($ ) $\mathrm{N}(\quad)$

- língua protruída $S(\quad) \quad N(\quad)$

- abertura de mandíbula adequada ( ) restrita ( ) exagerada ( )

- Deglutição:

- lábios ocluídos S( ) N( )

- munching $S(\quad) N(\quad)$

- regurgitação nasal $S(\quad) \quad N(\quad)$

- ejeção adequada $S(\quad) N(\quad)$

- escape extra-oral S( ) N( )

- resíduos após deglutição $S($ ) N ( )

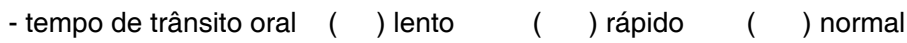

- sem tosse ( )

- com tosse: ( ) antes deglutição ( ) após deglutição

- sinais sugestivo de aspiração $S($ ) N ( ) Quais:

- manobras utilizadas:

PASTOSO:

- Captação:

- lábios ocluídos $\mathrm{S}($ ) $\mathrm{N}($ )

- língua protruída $S(\quad) \quad N(\quad)$

- abertura de mandíbula adequada ( ) restrita ( ) exagerada ( )

- Deglutição:

- lábios ocluídos $S(\quad) \quad N(\quad)$

- munching $S(\quad) N(\quad)$

- regurgitação nasal $S(\quad) \quad N(\quad)$

- escape extra-oral S( ) N( )

- tempo de trânsito oral ( ) lento

- sem tosse ( )

- sinais sugestivo de aspiração $S($ ) N ( ) Quais:

- manobras utilizadas:

Figura 1 - Protocolo de avaliação funcional 
Tabela 1 - Avaliação funcional da deglutição antes da intervenção fonoaudiológica na consistência líquida

\begin{tabular}{lccc}
\hline & Categoria & N & Percentual \\
\hline Captação: & & & 35 \\
\hline Lábios ocluídos & Sim & 7 & 65 \\
Língua protruída & Não & 13 & 70 \\
& Sim & 14 & 30 \\
\hline Deglutição: & Não & 6 & 35 \\
\hline Lábios ocluídos & Sim & 7 & 65 \\
Munching & Não & 13 & 65 \\
& Sim & 13 & 35 \\
Regurgitação Nasal & Não & 7 & 0 \\
& Sim & 0 & 100 \\
Escape extra-oral & Não & 20 & 65 \\
Língua protruída & Sim & 13 & 35 \\
& Não & 7 & 70 \\
Ejeção adequada & Sim & 14 & 30 \\
Sinais sugestivos de aspiração & Não & 6 & 60 \\
& Sim & 12 & 40 \\
& Não & 8 & 35 \\
\hline
\end{tabular}

$\mathrm{n}=$ número

Tabela 2 - Avaliação funcional da deglutição antes da intervenção fonoaudiológica na consistência pastosa

\begin{tabular}{lccc}
\hline & Categoria & $\mathbf{n}$ & Percentual \\
\hline Captação: & & & 35 \\
\hline Lábios ocluídos & Sim & 7 & 65 \\
Língua protruída & Não & 13 & 50 \\
& Sim & 10 & 50 \\
\hline Deglutição: & Não & 10 & 30 \\
\hline Lábios ocluídos & Sim & & 70 \\
Munching & Não & 6 & 65 \\
Regurgitação Nasal & Sim & 14 & 35 \\
& Não & 13 & 0 \\
Escape extra-oral & Sim & 7 & 100 \\
& Não & 0 & 55 \\
Língua protruída & Sim & 20 & 45 \\
& Não & 11 & 60 \\
Ejeção adequada & Sim & 9 & 40 \\
Sinais sugestivos de aspiração & Não & 12 & 60 \\
& Sim & 8 & 40 \\
& Não & 12 & 35 \\
\hline
\end{tabular}

$\mathrm{n}=$ número 
Tabela 3 - Avaliação funcional da deglutição após intervenção fonoaudiológica na consistência líquida

\begin{tabular}{lccc}
\hline & Categoria & $\mathbf{n}$ & Percentual \\
\hline Captação: & & & 45 \\
\hline Lábios ocluídos & Sim & 9 & 55 \\
Língua protruída & Não & 11 & 65 \\
& Sim & 13 & 35 \\
\hline Deglutição: & Não & 7 & 45 \\
\hline Lábios ocluídos & Sim & 9 & 55 \\
Munching & Não & 11 & 65 \\
& Sim & 13 & 35 \\
Regurgitação Nasal & Não & 7 & 0 \\
& Sim & 0 & 100 \\
Escape extra-oral & Não & 20 & 50 \\
Língua protruída & Sim & 10 & 50 \\
& Não & 10 & 60 \\
Ejeção adequada & Sim & 12 & 40 \\
Sinais sugestivos de aspiração & Não & 8 & 60 \\
& Sim & 12 & 40 \\
& Não & 8 & 30 \\
\hline
\end{tabular}

$\mathrm{n}$ = número

Tabela 4 - Avaliação funcional da deglutição após intervenção fonoaudiológica na consistência pastosa

\begin{tabular}{lccc}
\hline & Categoria & N & Percentual \\
\hline Captação: & & & 50 \\
\hline Lábios ocluídos & Sim & 10 & 50 \\
Língua protruída & Não & 10 & 50 \\
& Sim & 10 & 50 \\
\hline Deglutição: & Não & 10 & 45 \\
\hline Lábios ocluídos & Sim & & 55 \\
Mastigação fásica & Não & 9 & 55 \\
& Sim & 11 & 45 \\
Regurgitação Nasal & Não & 11 & 0 \\
& Sim & 9 & 100 \\
Escape extra-oral & Não & 0 & 40 \\
Língua protruída & Sim & 20 & 60 \\
& Não & 8 & 50 \\
Ejeção adequada & Sim & 12 & 50 \\
Sinais sugestivos de aspiração & Não & 10 & 60 \\
& Sim & 10 & 40 \\
& Não & 12 & 30 \\
& Sim & 8 & 70 \\
\hline
\end{tabular}

$\mathrm{n}=$ número 


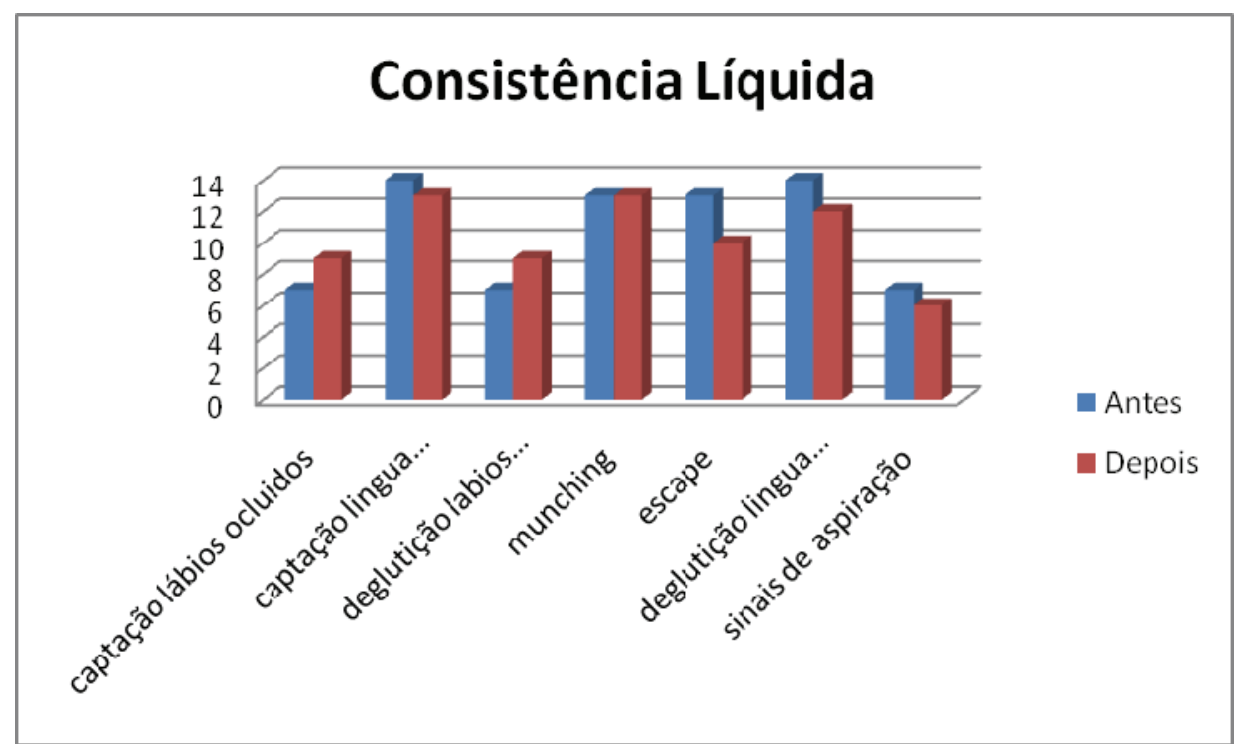

Figura 2 - Comparação dos dados obtidos na consistência líquida antes e após fonoterapia

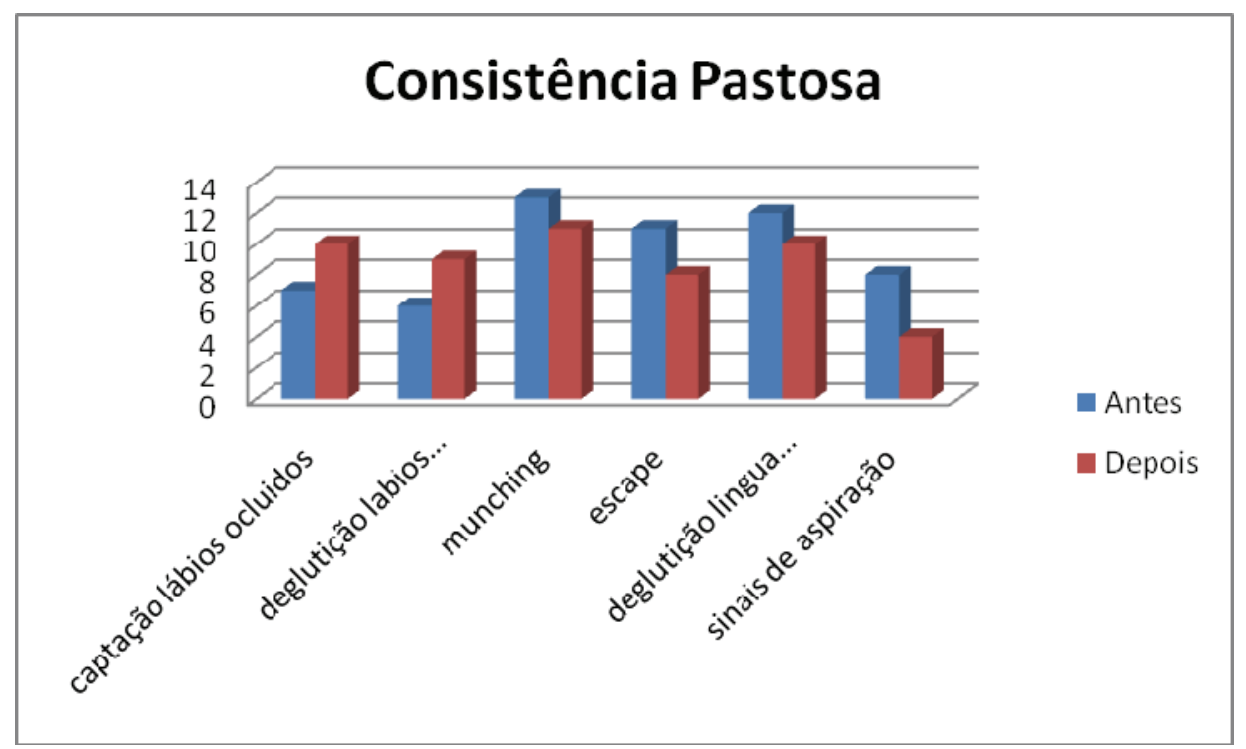

Figura 3 - Comparação dos dados obtidos na consistência pastosa antes e após fonoterapia

\section{DISCUSSÃO}

A alimentação é uma função vital para o ser humano. É um dos primeiros contatos e estabelecimento da relação mãe/filho. Está muito ligada ao convívio e interação social.

Os problemas alimentares e nutricionais da infância freqüentemente apresentam as disfagias como etiologia principal e estão associados à alteração do desenvolvimento e de saúde ${ }^{16}$.

A fundamentação metodológica de avaliação permite que os dados sejam coletados, de maneira pré-estabelecida, possibilitando análise e definição de condutas ${ }^{17}$.

A mastigação é uma das funções mais importantes do sistema estomatognático ${ }^{18}$, porém devido à grande dificuldade motora dos pacientes atendidos não foi possível realizar a avaliação com alimentos sólidos e, conseqüentemente, a mastigação.

A persistência do movimento de sucção com a língua nas crianças com PC, além de dificultar a aquisição de movimentos mais adequados de língua, quando acompanhados de hiper ou 
hipossensibilidade oral, também conduz a dificuldade de mastigar e deglutir alimentos ${ }^{11}$.

Estudar a eficácia da fonoterapia para disfagia em crianças com distúrbios neurológicos é tarefa complexa devido à quantidade de sintomas associados que apresentam ${ }^{5,19-20}$.

O desenvolvimento motor evolui de forma organizada. Após o $4^{\circ}$ ou $5^{\circ}$ mês de vida, a condição reflexa é substituída pela movimentação oral voluntária para alimentação, tornando-se assim mais efetiva com a estabilidade da cabeça, melhorando o controle da mandíbula que é influenciada pelo alinhamento do tronco o qual depende da estabilidade da área pélvica ${ }^{21}$. Por isso, a nossa orientação aos cuidadores sobre como posturar o paciente na hora da alimentação faz-se extremamente necessária. Crianças com desordens sensório-motoras necessitam de adequado controle para êxito na alimentação ${ }^{22-23}$.

Os padrões motores orais dos portadores de PC são muito específicos e variam de indivíduo para indivíduo ${ }^{24}$.

A disfagia tem sido uma preocupação mundial ${ }^{25-27}$. A PC é freqüentemente associada com distúrbios de deglutição $28-31$. Em um estudo ${ }^{32}$ foi analisado que $24 \%$ dos pacientes apresentavam penetração laríngea de alimentos e $39 \%$ aspiração silente de saliva. Desses pacientes, mais da metade não apresentavam proteção das vias aéreas pelo reflexo de tosse.

À medida que a criança com hipotonia e padrão extensor anormal tenta levantar e rodar a cabeça ela necessita fixar-se e então utiliza os extensores da cabeça e os músculos extrínsecos da língua para estabilizar-se. Isto terá como resultado hiperextensão anormal da cabeça, pescoço e também retração da língua; isto é, muitas vezes o primeiro problema que dificultará a alimentação da criança com PC. Com apresentação de líquidos ou semisólidos irá ocorrer maior extensão da cabeça e pescoço. Para controlar a comida na cavidade oral poderão aparecer movimentos orais compensatórios, tais como: protrusão de lábios e mandíbula, munching, protrusão lingual, pressão lingual anterior acompanhada de abertura e protrusão labial. A eficiência da sucção poderá ser pobre levando a um consumo nutricional insuficiente. Haverá também incoordenação da sucção-deglutição-respiração que será percebida com episódios de engasgos e tosse durante a alimentação ${ }^{33}$. Na população avaliada na ABBR foram encontrados dados que confirmam as observações descritas acima.

Nas tabelas apresentadas neste estudo antes e após intervenção fonoaudiológica, observou-se que pacientes com PC se beneficiam das orientações aos responsáveis, manobras facilitadoras e exercícios miofuncionais.
Os responsáveis pelos pacientes foram de extrema importância, seguindo as orientações recebidas, mantendo a freqüência ao tratamento e continuando o trabalho orientado em casa.

$\mathrm{Em}$ todos os casos reavaliados percebeu-se uma melhora, mesmo que discreta do padrão de deglutição, gerando assim uma melhora global em seu padrão de alimentação. Porém, o tempo de atendimento utilizado nessa pesquisa (três meses), mostrou-se reduzido para a realização de fonoterapia e orientação. $\mathrm{Na}$ literatura foram encontrados dados que corroboram esse achado. Em um estudo ${ }^{11}$ a média de atendimento foi de dez meses e em outro ${ }^{5}$ o tempo também foi de três meses, e o autor não achou significativo para maiores adequações nos quadros de disfagia.

Pacientes com déficits neurológicos podem possuir incoordenação entre respiração e deglutição, permitindo a aspiração durante a deglutição, bem como a inspiração iniciando-se logo antes da deglutição. A primeira linha de defesa das vias aéreas contra a aspiração é a própria função normal da laringe durante a deglutição, que inclui a elevação e anteriorização da laringe, a retroversão da epiglote e o fechamento glótico. O esfíncter esofageano superior, sempre contraído, impede também que material de refluxo penetre na faringe e seja aspirado. No entanto, se houver aspiração de material para a traquéia, a tosse é o primeiro mecanismo de defesa ${ }^{34}$.

As desordens da função motora oral podem gerar um espectro de incapacidades em cada etapa do processo de deglutição com potenciais interações simultâneas. Em um número significativo de crianças, as alterações crônicas da deglutição resultam em desnutrição, desidratação, aspiração e pneumonia. Os sintomas usualmente observados nesses casos são regurgitação, dificuldade de deglutir a própria saliva, tosse no momento da alimentação e instabilidade respiratória ou apnéia, dentre outros, que podem gerar aversão para comer determinados alimentos, grande tempo despendido com alimentação e a necessidade de dieta especial, diferente da utilizada pelo restante da família. Estes sintomas acabam gerando uma situação de tensão e ansiedade nos pais, o que torna o ato de comer difícil, cansativo e de pouco prazer ${ }^{35}$.

Devido à importância do reflexo de tosse para a defesa da entrada de alimentos para traquéia, esse tópico está sempre presente nas avaliações de disfagia e na observação do fonoaudiólogo durante atendimento e orientação aos cuidadores. Vale à pena ressaltar a importância do trabalho em conjunto com a fisioterapia respiratória para desenvolvimento da tosse efetiva (eficaz para higienização das vias aéreas), o que muito nos ajuda no trabalho de reabilitação das disfagias. 
Nessa pesquisa, antes da intervenção fonoaudiológica dez pacientes (50\%) apresentavam episódios de tosse durante alimentação, como sinal sugestivo de aspiração. Após os três meses de tratamento esse número foi reduzido para seis (30\%).

O levantamento da incidência da disfagia em pacientes com PC permite, não apenas o conhecimento da doença e suas complicações, mas também alertar a equipe médica da necessidade de um acompanhamento mais detalhado e periódico relacionado à deglutição nesses $\operatorname{casos}^{36}$.

Discussões recentes sobre a eficácia da reabilitação em disfagia retratam evidências da atuação fonoaudiológica, porém discutem a necessidade de novas pesquisas sobre essa temática ${ }^{37}$. No caso desse estudo, foi percebido que o tempo de terapia (três meses) não foi suficiente para uma melhora mais efetiva na adequação do padrão de deglutição.

Há necessidade clara de mais pesquisas que estudem o impacto da reabilitação da deglutição e o uso de técnicas específicas 5 .

\section{CONCLUSÃO}

O presente estudo mostrou que a intervenção fonoaudiológica aos pacientes portadores de Paralisia Cerebral traz como resultado melhorias em seu padrão de alimentação, apesar do curto período de atendimento (três meses).

\begin{abstract}
Purpose: to analyze swallowing patterns before and after speech therapy intervention in a group of children with Cerebral Palsy who have dysphagia. Method: cross-sectional study, consisting of twenty children with Cerebral Palsy, aged between one and eight years, both genders. We used a clinical history with the family heads and the patients were subjected to functional swallowing assessment before and after speech intervention in a period of three months. Results: slight improvement was noted in the swallowing pattern which consequently brought a better pattern of meals to the patients. Conclusion: this study found that the speech intervention in patients with Cerebral Palsy brings as a result an improvement in their usual diet, despite the short treatment period(three months).
\end{abstract}

KEYWORDS: Cerebral Palsy; Speech Therapy; Feeding; Children

\section{REFERÊNCIAS}

1. Piovesana AMSG. Paralisia Cerebral: Contribuições do Estudo por Imagem. In: Souza AMC, Ferraretto I. Paralisia Cerebral - Aspectos Práticos. São Paulo: Frôntis Editorial; 1998, p. 9- 37. 2. Baladi ABPT, Castro NMD, Filho MCM. Paralisia Cerebral. In: Fernandes AC, Ramos ACR, Casalis MEP, Hebert SK, organizadores. AACD Medicina e Reabilitação - Princípios e Prática. São Paulo: Artes Médicas; 2007, p. 15-35.

3. Miyadahira S, Francisco RPV, Zugaib M. Quais os exames que podem relacionar a asfixia no parto e a paralisia cerebral? Rev. Assoc. Med. Bras. 2006; 52(3): 131.

4. Miranda S., Disfagias Neurológicas da Infância. In Costa, MMB, Castro LP. Tópicos em Deglutição e Disfagia. Rio de Janeiro. Ed MEDSI; 2003, p. 225-31.

5. Furkim AM, Sacco ABF. Eficácia da fonoterapia em disfagia neurogênica usando a escala funcional de ingestão por via oral (FOIS) como marcador. Rev CEFAC.2008; 10(4): 503-12.
6. Silva RG, Jorge AG, Peres FM, Cola PC, Gatto AR. Protocolo para controle de eficácia neurogênica (PROCEDON). Rev. CEFAC. 2010: 12(1): 75-81.

7. Cabre M, Serra-Prat M, Palomera E, Almirall J, Pallares $R$, Clavé P. Prevalence and prognostic implications of disphagia in elderly patients with pneumonia. Age Ageing. 2010: 39 (1): 39-45.

8. Santini CS. Disfagia Neurogênica. In: Furkim AM, Santini CS, organizadores. Disfagia Orofaríngeas. Carapicuíba: Pró-fono: 2001. p. 19-34.

9. Clavé P, De Kraa M, Arreola V, Girvent M, Farré $R$, Palomera $E$, et $A L$.The effect of bolus viscosity on swallowing function in neurogenic dysphagia. Aliment Pharmacol Ther. 2006 Nov; 24 (9): 1385-94.

10. Frazão YS. Disfagia na Paralisia Cerebral. In: Ferreiro LP, Lopes DMB, Limongi SCO, organizadores. Tratado de Fonoaudiologia. São Paulo: Roca; 2004, P 370-85.

11. Silvério CC, Henrique CS. Indicadores da evolução do paciente com paralisia cerebral e disfagia orofaríngea após intervenção terapêutica. Rev Soc Bras Fonoaudiologia. 2009; 14(3); 381-6. 
12. Silva MC, Carvalho MLV, Nemr K, Marchesan IQ. Frênulo de língua alterado e interferência na mastigação. Rev CEFAC. 2009; 11(3): 363-9.

13. Limongi, SCO. Avaliação e terapia fonoaudiológica do paralítico cerebral. In:. Tabith Jr A. Foniatria - Disfonias, Fissuras Labiopalatais, Paralisia Cerebral. São Paulo: Cortez Editora; 1984, p. 77-117.

14. Mueller H. Alimentação. In: Finnie NA. O manuseio em casa da criança com paralisia cerebral. São Paulo. Editora Malone; 1980, p.131-53.

15. Crickmay, MC. Logopedia y el enfoque Bobath in paralisis cerebral. Buenos Aires. Editora Panamericana; 1977. P.89-110.

16. Marrara JL, Duca AP, Dantas RO, Trawitzki LVV, Lima RAC, Pereira JC. Deglutição em crianças com alterações neurológicas: avaliação clínica e videofluoroscopia. Rev. Pro-fono. 2008; 20(4) 231-6.

17. Padovani AR, Moraes DP, Mangili LD, Andrade CRF. Protocolo fonoaudiológico de avaliação do risco de disfagia (PARD). Rev Soc. Bras. Fonoaudiologia. 2007; 12(3) 199-205.

18. Lima RMF, Freire OCP, Nepomuceno Filho JL, Stampford S, Cunha DA, Silva HJ. Padrão mastigatório em crianças de 5 a 7 anos: suas relações com o crescimento craniofacial e hábitos alimentares. Rev. CEFAC. 2006; 8(2): 205-15.

19. Cesa CC, Ecco CT, Bersch R, Chiappetta ALML. Funções do sistema estomatognático e reflexos motores orais em crianças com encefalopatia crônica infantil do tipo quadriparesia espástica. Rev. CEFAC. 2004; 6(2): 158-63.

20. Gomes AM, Novaes CRG, Assencio-Ferreira VJ. Disfagia orofaríngea neurogênica. Principais fatores determinantes da recusa alimentar no paciente portador de paralisia cerebral do tipo espástica institucionalizado. Rev. CEFAC. 2002; 4 : 25-8.

21. Telles MS, Macedo CS. Relação entre desenvolvimento motor corporal e aquisição de habilidades orais. Rev. Pro-fono. 2008; 20(2); 117-22.

22. Wolf LS, Glass RP. Feeding and swallowing disorderes in infancy: assessment and management. Therapy skill builderes a division of communication skill buideres. Arizona. 1992.

http://dx.doi.org/10.1590/S1516-18462011005000057

RECEBIDO EM: 15/06/2010

ACEITO EM: 11/01/2011

Endereço para correspondência

Cláudia Inês Oliveira Vianna

Rua Jardim Botânico, 660 - Jardim Botânico

Rio de Janeiro - RJ

CEP: 22461-000

E-mail: claudiaiovianna@uol.com
23. Readstone F. West JF. The importance postural control for feeding. Pediatric Nussing Journal. 2004; 30(2): 97-100.

24. Gianni Mac. Paralisia Cerebral - Aspectos Clínicos. In Moura EW, Silva PAC. Fisioterapia: Aspectos Clínicos e Práticos de Reabilitação. São Paulo: artes Médicas; 2005, p. 13.

25. Costa BAK, Azevedo EH, Vartanjan JG, Nishimoto IN, Kowaslki LP, Carrara de Angelis E. Quality of life relates to swallowing after tongue câncer treatment. Dysphagia. 2008; 23(2): 189-92.

26. Khaldoun E, Woisard V, Verin E. Validation in French of the SWAL-QOL scale in patients with oropharungeal dysphagia. Gastroenterol Clin Biol. 2009; 33(3):167-71.

27. Bogaardt HC, Speyer R, Baijens LW, Fokkens WJ. Cross-cultural adaptation and validation of the Dutch version of SWAL-QOL. Dysphagia. 2009; 24(1): 66-70.

28. Drent LV. Pinto EALC. Problemas de alimentação em crianças com doença do refluxo gastroesofágico. Rev. Pro-fono. 2007; 19(1): 59-66. 29. Haak P. Lenski M. Hidecker MJ. Li M. Paneth N. Cerebral Palsy and aging. Dev. Med. Child Neurol. 2009; 51(4): 16-23

30. Baladin S. Hemsley B. Hanley L. Sheppard JJ. Understanding mealtime changes for adults with cerebral palsy and implications for support services. J Intellect Dev Disabil. 2009; 34(3); 197-206.

31. Furkim AM. Behlau MS. Wecky LLM. Avaliação clínica e videofluoroscópica da deglutição em crianças com paralisia cerebral tetraparética espástica. Arq. Neuropsiquiatria. 2003; 61 (3); 611-6.

32. Bader CA. Niemann G. Dhysphagia in children with cerebral palsy - fiberoptic - endoscopicfindings. 2010; 89(2); 90-4

33. Frazão YS, Furkim AM. Disfagia na Paralisia Cerebral do Tipo Tetraparético. In Costa, MMB., Castro LP. Tópicos em Deglutição e Disfagia. Rio de Janeiro. Ed MEDSI; 2003, p.257-63.

34. Isola AM. Pneumologia. In: Fernandes AC, Ramos ACR, Casalis MEP, Hebert SK, organizadores. AACD Medicina e Reabilitação Princípios e Prática. São Paulo: Artes Médicas; 2007, p. 364-75.

35. Filho EDM, Genaro KF, Aurélio SR. Análise comparativa dos padrões de deglutição de crianças com paralisia cerebral e crianças normais. Revista Brasileira de otorrinolaringologia. 2002; 68(2): 167-73.

36. Lucchi C, Florio CPF, Silverio CC, Reis TM. Incidência de disfagia orofaríngea em pacientes com paralisia cerebral do tipo tertraparéticos espásticos institucionalizados. Rev. Soc. Bras. Fonoaudiologia. 2009; 14(2): 172-6.

37. Logemann JA. Treatment of oral and pharyngeal. Phys Med Rehabil Clin N Am. 2008; 19 (4): 803-16. 